\title{
Simulating Solar and Astrophysical Plasmas in Laboratory Experiments and New Models Motivated by these Experiments
}

\author{
P. M. Bellan, S. C. Hsu*, J. F. Hansen**, S. K. Tripathi, S. You, \\ and S. E. Pracko \\ MC 128-95, Caltech, Pasadena CA 91125, USA \\ ${ }^{*}$ now at $L A N L,{ }^{*}$ now at $L L N L$
}

\begin{abstract}
Laboratory plasma configurations have been developed which simulate solar coronal loops and astrophysical jets. Observed phenomena include the magnetic pinch effect, magnetic hoop force, restraining of the hoop force by an optional externally applied "strapping" magnetic field, MHD-driven jets and their collimation via the stagnation of convected magnetic flux, magnetic kinking, co- and counter helicity merging of two distinct magnetic structures, enhanced x-ray production upon merging, magnetic reconnection and formation of detached structures. These observations have motivated a model which invokes jet collimation to explain the observed collimation of solar coronal loops.
\end{abstract}

\section{INTRODUCTION}

Using specially designed experiments we have recently been able to create controlled, reproducible laboratory simulations of both solar coronal loops and astrophysical jets. Pulsed power technology, originally developed for spheromak fusion devices [1], is used in these experiments to achieve relevant MHD-dominated plasma regimes having large Lundquist number and small $\beta$ while imposed magnetic, electric, and mass flux boundary conditions approximate the respective boundary conditions of coronal loops and astrophysical jets.

The experiments effectively take place in a half-space $-\infty<x, y<\infty$ and $0<z<\infty$ with boundary conditions imposed on physical quantities in the $z=0$ plane. The boundary conditions involve specification of the tangential electric field $\mathbf{E}_{t}$ and of $B_{z}, J_{z}$, and $\rho U_{z}$ in the $z=0$ plane. Adjustable external coils prescribe $B_{z}$, adjustable capacitor banks and electrode design prescribe $J_{z}$ and $\mathbf{E}_{t}$, and adjustable puffed gas valves prescribe $\rho U_{z}$.

A useful starting point for interpreting the experiments is the Woltjer-Taylor relaxation model. This model ignores dynamics, assumes zero $\beta$, and predicts that MHD instabilities cause plasmas to relax to a force-free state,

$$
\nabla \times \mathbf{B}=\alpha \mathbf{B}
$$

CP703, Plasmas in the Laboratory and in the Universe, edited by G. Bertin, D. Farina, and R. Pozzoli (C) 2004 American Institute of Physics 0-7354-0176-4/04/\$22.00 
where $\alpha$ is spatially uniform. Our experiments indicate that, while the WoltjerTaylor model is a helpful starting point for understanding, the effect of nonequilibrium dynamics, compressibility, small but finite $\beta$, and $\alpha$ gradients cause actual behavior to be considerably more complex.

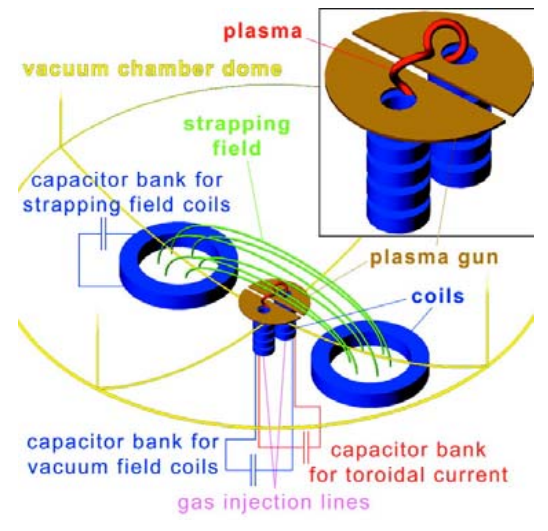

FIGURE 1. Insert (top right): Basic layout of coronal loop simulation experiment. Distance between footpoints is $8 \mathrm{~cm}$. Main figure: Optional large additional coil pair provides adjustable strapping field. The strapping field counteracts the hoop force which drives the expansion of major radius shown in Fig.2.
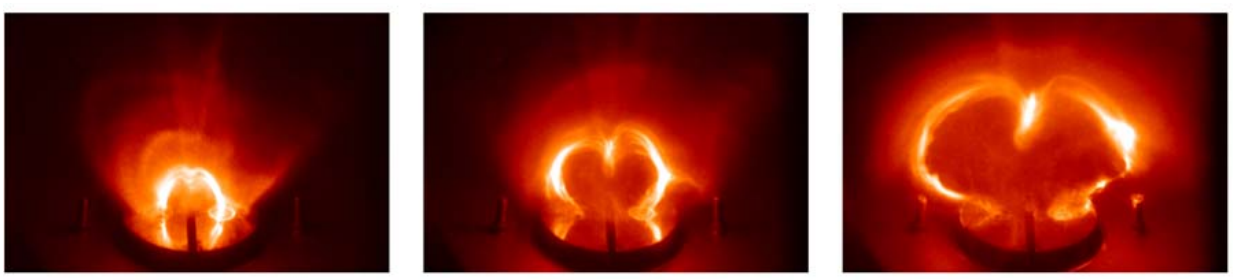

FIGURE 2. Simulated coronal loop at three successive times: hoop force causes loop major radius to expand while kinking causes loop axis to become helical.

\section{MAIN RESULTS OF EXPERIMENTS}

\section{Solar Coronal Loops}

The insert at the top right of Fig.1 shows the basic experimental configuration $[3,4]$ for simulating solar coronal loops. A pair of small electromagnets are energized by a slow capacitor bank (10 ms quarter cycle) to produce an arch-shaped, potential magnetic field protruding into the end dome of the large vacuum chamber. Hydrogen gas is then puffed on a $100 \mu$ s time scale into the region between the magnet poles. Finally, a fast (10 $\mu$ s quarter cycle) high energy capacitor bank is discharged across D-shaped electrodes located slightly above the magnet poles. The applied voltage of several kilovolts quickly breaks down the gas to form plasma and then drives 30-50 kiloamps of electric current through the plasma. Driving this electric current along the magnetic field linking the magnet poles injects magnetic helicity into the volume at the rate $\dot{K}=2 V \Phi$ where $V$ is the electrostatic potential 
of the electrode and $\Phi$ is the magnetic flux intercepting the electrode [2]. The characteristic time for the dynamical evolution of the experiments is $\sim 1 \mu$ s which is slow compared to the characteristic Alfvén time, but fast compared to the resistive diffusion time. The combination of imposed current $I$ and magnetic flux $\psi$ at an electrode determines $\alpha=\mu_{0} I / \psi$ as seen by integrating Eq.(1) over the electrode surface. Reproducible measurements indicate that:

1. The simulated coronal loop quickly develops a nearly uniform cross-section along its length; see Fig. 2(left). This axial uniformity (filamentary structure) is similar to observations of actual solar coronal loops [5, 6] and is in marked contrast to the $\alpha \simeq 0$ situation where the arched flux tube has a non-uniform cross-section characteristic of potential magnetic fields.

2. The loop axis writhes such that, when viewed from above, its projection onto the ground plane has an $S$-shape (sigmoid). The side view is the projection of a helix about a semi-circular axis [see Fig. 2(middle, right)].

3. The major radius of the simulated coronal loop continuously expands (see Fig. 2 sequence) because of the so-called hoop force resulting from the mutual repulsion of current segments on opposite sides of a current loop. The hoop force, proportional to $I$, tries to expand the loop major radius whereas the field line tension, proportional to $\psi$, tries to keep the field line geometry in its initial potential configuration. The outcome of the "tug-of-war" between hoop force and field line tension determines how much the loop expands.

By adding another set of coils arranged as in Fig. 1 to make an additional "strapping" field $\mathbf{B}_{\text {ext }}$, the hoop force can be completely counteracted by the downward $\mathbf{J} \times \mathbf{B}_{\text {ext }}$ force resulting from the interaction between the loop current and the strapping field [3].

\section{Merging of Two Adjacent Coronal Loops}

In this set of experiments $[4,7]$ two adjacent coronal loops are created, each similar to the single loop shown in Fig. 2. The axial currents of the two loops have the same sense, but the axial magnetic fields have either the same or opposite sense. The loops mutually attract because their axial currents are parallel, but their respective helicities are of either the same or opposite polarity (co- or counterhelicity) depending on the axial magnetic field polarities.

In co-helicity merging, helicity is observed to transfer from one loop to its neighbor causing the receiving loop to erupt faster than the donor loop. In counterhelicity merging, the axial magnetic field is annihilated while the total current is conserved. This destroys the field line tension opposing the hoop force and leads to an observed tendency towards overall faster expansion (eruption) of the merged configurations at low axial field strengths where the field line tension is small to begin with. Also observed in counter-helicity merging are a more than an order of magnitude increase in soft x-ray emission, a sharp onset of this increase, and a localized bright region at the top of the loops that appears to be associated with the enhanced soft x-ray emission. 

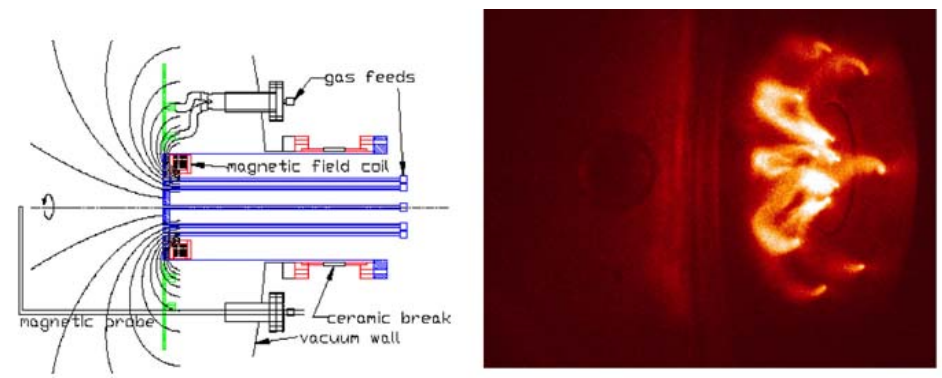

FIGURE 3. Left: Experimental layout showing poloidal magnetic field produced by coil just to right of plane of disk (blue) and annulus (green) electrodes. Right: Set of eight loops spanning from gas nozzles on disk electrode to gas nozzles on annulus electrode; these loops form immediately after breakdown and the loop axes follow the initial potential poloidal magnetic field.
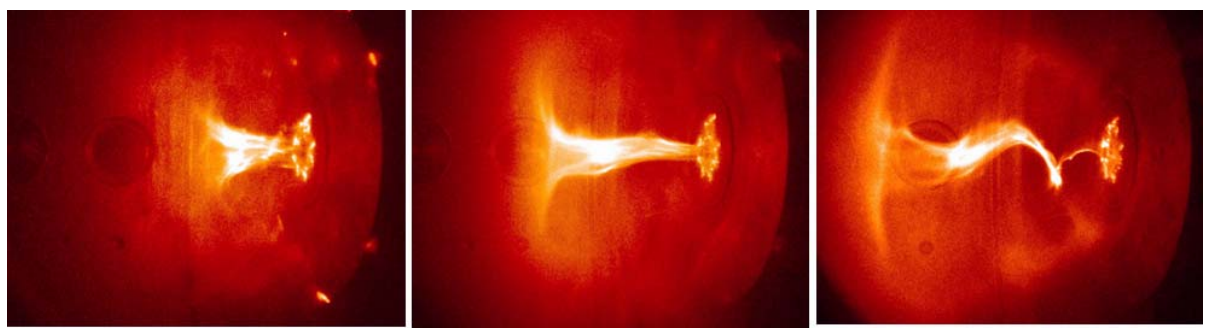

FIGURE 4. Left: Inboard portions of eight loops coalesce to form central column. Middle: Jet-like axial expansion of central column. Right: Kink instability of central column when it reaches a critical length.

\section{Astrophysical Jet Simulation}

The astrophysical jet simulation experiment $[8,9]$ involves similar laboratory methods but differs geometrically by having a completely azimuthal source. As shown in Fig. 3(left), one electrode is a circular disk while the other is a surrounding co-planar annulus separated from the disk by a small radial gap. A coil located behind the gap creates a poloidal magnetic field which links the disk and annulus. The topology of this coil-produced poloidal field is outlined in Fig. 3(right) by initial discrete plasma loops following this field. The geometry simulates a rotating accretion disk intercepting poloidal magnetic field produced by a central magnetized object such as a star, active galactic nucleus, or black hole. Rather than rotating the annulus like an accretion disk to create a radial electric field $E_{r}=-U_{\phi} B_{z}$, here we simply apply a potential difference between the disk and annulus. The radial electric field $E_{r}$ associated with the potential difference drives a poloidal current essentially similar to the current driven in the astrophysical situation. The interaction between the poloidal current and its self-generated toroidal magnetic field produces MHD forces which accelerate and collimate the jet. 
Five distinct steps are observed: (i) creation of a set of discrete, collimated, current-carrying loops consisting of plasma ingested from the localized gas sources; these initial loops trace out the shape of the initial vacuum poloidal field [see Fig. 3(right)], (ii) merging of the inboard portions of these loops to form an axisymmetric central column [see Fig. 4(left)], (iii) jet-like expansion of the axisymmetric central column [see Fig. 4(middle)], (iv) kink instability[8] of the central column [see Fig. 4(right)] when the column becomes sufficiently long to satisfy the $q=1$ kink instability condition, (v) conversion [9] of toroidal flux into poloidal flux because the helical current channel constitutes a solenoid. For sufficiently large current, the kinked current channel disconnects from the source and forms a detached plasmoid (spheromak). The mass flux boundary condition on the source surface is found to be critical: jets do not form if the gas valves are turned off, thereby removing plasma needed for jet ingestion.

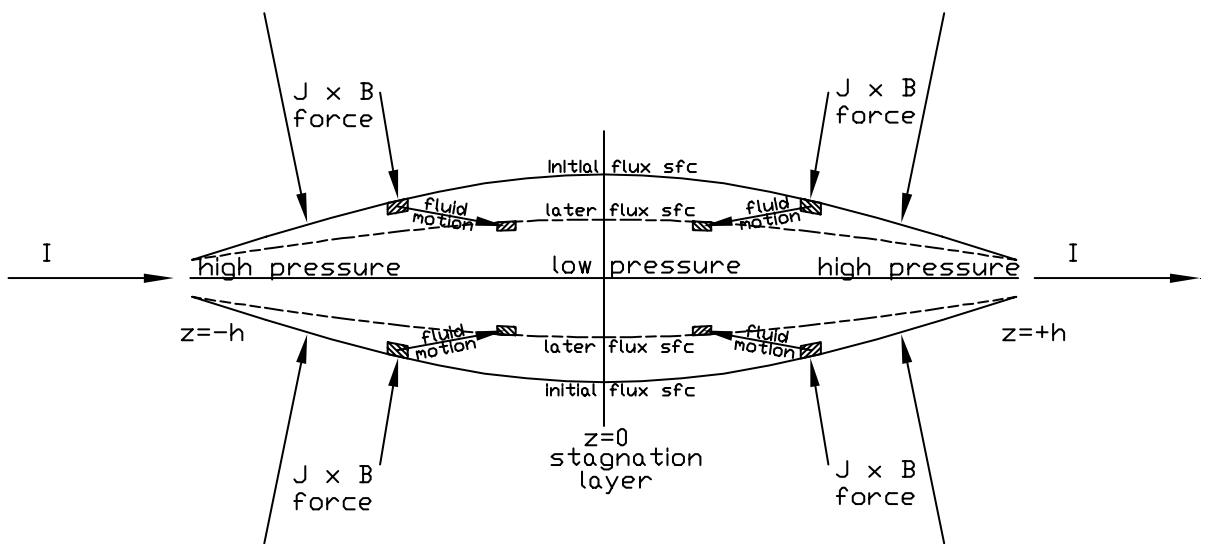

FIGURE 5. Schematic of poloidal flow of plasma which is constrained to stay on constant $\psi$ surfaces and which stagnates at $z=0$. Flow is driven by $z$-component of $\mathbf{J} \times \mathbf{B}$ force and is from $z= \pm h$ towards $z=0$.

\section{MODEL EXPLAINING CORONAL LOOP COLLIMATION}

This model [10] argues that bidirectional converging jet-like flows should occur in a current-carrying coronal loop and that the pile-up of the magnetic flux convected by these flows causes the strong collimation typically observed $[5,6]$ in coronal loops. The model uses the axisymmetric cylindrical geometry representation shown in Fig. 5 so that the loop axis is the $z$-axis, the footpoints are at $z= \pm h$ and the loop apex corresponds to $z=0$. Flux coordinates are used such that $\psi(r, z)$ and $I(r, z)$ are respectively the poloidal flux and the poloidal current linked by a circle of radius $r$ at axial location $z$; the magnetic field is given as $\mathbf{B}=(2 \pi)^{-1}\left(\nabla \psi \times \nabla \phi+\mu_{0} I \nabla \phi\right)$ where $\phi$ is called the toroidal angle. Driving current $I$ along an initially bulged 
(i.e., non-collimated) flux tube results in three distinct stages [10]:

The first stage is fast and consists of a twisting of the magnetic field about the $z$ axis. An associated transient toroidal plasma velocity $U_{\phi}$ causes the plasma to rotate in frozen-in fashion with the twisting magnetic field lines. This stage is incompressible and does not change $\psi(r, z)$ so that the flux tube maintains its initial bulged, axially non-uniform poloidal profile. The velocity $U_{\phi}$ is proportional to $z \partial I / \partial t$ and the toroidal acceleration is proportional to $z \partial^{2} I / \partial t^{2}$ so that $U_{\phi}$ vanishes when $I$ becomes steady-state. The velocity $U_{\phi}$ is identified as the polarization drift resulting from transient polarization electric fields.

The second stage has steady-state $I$ but is not in equilibrium because of unbalanced MHD forces. These forces drive slow poloidal plasma flows from $z= \pm h$ towards $z=0$, i.e., from where the flux tube diameter is small to where the diameter is large. Specifically, the flows are driven by $(\mathbf{J} \times \mathbf{B})_{z}$, a force proportional to $-\partial I^{2} / \partial z$. Thus, unlike the first stage where motion is toroidal and results from a temporal derivative of $I$, here the motion is poloidal and results from a spatial derivative of $I$.

In the third stage, the converging flows stagnate at $z=0$ and thermalize. Since toroidal flux is frozen into the flow, toroidal flux convected from $z= \pm h$ to $z=0$ piles up at $z=0$ and so increases the toroidal magnetic flux density at $z=0$. Thus $B_{\phi}$ increases at $z=0$ and since $I$ is constant, Ampere's law $2 \pi r B_{\phi}=\mu_{0} I$ dictates that $r$, the flux tube radius, must decrease. The flux tube thus approaches axial uniformity (collimation) and also is loaded with ingested plasma heated by flow thermalization at the $z=0$ stagnation layer. This collimation is similar to that of an astrophysical jet but has mirror symmetry about the $z=0$ plane.

Figure 5 sketches this process and shows how the $(\mathbf{J} \times \mathbf{B})_{z}$ force pushes plasma from both ends to the middle in a flux-conserving manner and how the pile-up of toroidal flux at $z=0$ leads to collimation.

\section{ACKNOWLEDGMENTS}

Supported by USDOE grants DE-FG03-97ER54438 and DE-FG03-98ER54461.

\section{REFERENCES}

1. P. M. Bellan, Spheromaks (Imperial College Press, London, 2000).

2. P. M. Bellan, "Magnetic helicity and relaxation: theory" in AGU Monograph 111 "Helicity of the Photospheric Magnetic Field" (eds: M. R. Brown, R. C. Canfield, and A. A. Pevtsov, June 1999), p. 119.

3. J. F. Hansen and P. M. Bellan, Astrophys. J. 563, L183 (2001).

4. J. F. Hansen, Ph.D. thesis, Caltech, 2001.

5. J. A. Klimchuk, Solar Physics 193, 53 (2000).

6. M. J. Aschwanden, R. W. Nightingale, and D. Alexander, Astrophys. J. 541, 1059 (2000).

7. J. F. Hansen, S. K. Tripathi, and P. M. Bellan, to be submitted for publication, 2003.

8. S. C. Hsu and P. M. Bellan, Mon. Not. R. Astron. Soc. 334, 257 (2002).

9. S. C. Hsu and P. M. Bellan, Phys. Rev. Letters 90, article 215002 (2003).

10. P. M. Bellan, Phys. Plasmas 10 Pt 2, 1999 (2003). 